\title{
Vitamin D: evidence for an association with coronary collateral circulation development?
}

\author{
Jose Lima Jr' ${ }^{1}$, Vijay Kunadian ${ }^{1,2}$ \\ ${ }^{1}$ Institute of Cellular Medicine, Newcastle University, Newcastle upon Tyne, United Kingdom \\ ${ }^{2}$ Freeman Hospital, Newcastle upon Tyne NHS Foundation Trust, Newcastle upon Tyne, United Kingdom
}

Postep Kardiol Inter 2015; 11, 3 (41): 174-176

DOI: $10.5114 /$ pwki.2015.54008

Contrary to past beliefs that the human coronary artery system lacks arterial anastomoses, it is now well established that coronary arteries possess a complex network of collateral circulation. Indeed, humans present one of the most developed coronary collateral systems amongst mammals [1]. Coronary collateral vessels originate during embryogenesis by a mechanism known as vasculogenesis, in which local signals promote the migration and differentiation of endothelial progenitor cells, resulting in de novo synthesis of blood vessels. After the embryological stage, development of collaterals relies on two other processes, namely angiogenesis and arteriogenesis. In angiogenesis, new capillaries are formed by sprouting of endothelial cells from pre-existing mature vessels under conditions of ischemia in response to several growth factors, including the vascular endothelial growth factor (VEGF) and basic fibroblast growth factor (bFGF). Vascular smooth muscle cells and pericytes also play a role in this process, being particularly important for the stabilization and maturation of new vessels. In contrast, arteriogenesis is defined as a structural remodelling of collaterals pre-formed during embryogenesis, promoted by a redistribution of blood flow and increased shear forces in vessel walls. This is especially observed after coronary total occlusion, when a decrease in pressure distal to the site of obstruction redirects the flow to arteries outside the ischemic area, leading to a positive remodelling of pre-existing arterioles, which can increase up to twelve fold in size [2].

Although functioning collaterals are observed in approximately $20 \%$ to $25 \%$ of individuals without coronary artery disease (CAD), the degree of coronary obstruction is one of the main determinants of collateralization - individuals with CAD present high prevalence of collaterals. In fact, collateral functionality has been associated with the severity of CAD, as it has been shown that patients with chronic total coronary occlusion have a higher collateral flow index (CFI) than those without chronic occlusion [3]. Therefore, collateral circulation seems to play an important role in salvaging areas of myocardium under ischemia, as exemplified by the observation that well-developed collaterals can limit infarct size [4]. In terms of prognostic value, Seiler et al. [5] evaluated the impact of collateral function, as assessed quantitatively by CFI, on all-cause mortality in a cohort of 1,181 patients with CAD and observed an increased 15-year cumulative survival rate $(65 \%)$ in the group with higher CFI $(\geq 25)$ in comparison with the lower CFI $(<25)$ group (48\%), $p=$ 0.0057 . Furthermore, they suggested a causal relationship between well-functioning collaterals and lower mortality, as participants who had been enrolled in trials receiving arteriogenic therapy (granulocyte-macrophage colony stimulating factor; granulocyte colony stimulating factor; external counter-pulsation; or physical exercise) had higher cumulative 10-year survival rate (88\%) and CFI during follow-up than the placebo group (75\%), $p=0.0482$. These findings are similar to those of a meta-analysis, including 13 studies, which reported a relative risk reduction in all-cause mortality in participants with well-functioning coronary collateral circulation $\left(\mathrm{RR}=0.64 ; 95 \% \mathrm{Cl}: 0.45-0.91 ; p=0.0006 ; l^{2}=66.5 \%\right)[6]$. Thus, therapeutic strategies that aim to improve collateral circulation, especially by promoting arteriogenesis, could potentially improve patients' prognostic outcomes.

In this context, the effects of vitamin D on the vasculature have been increasingly studied and point towards an interesting regulatory role. Vitamin $D$ is a prohormone initially synthesized in the skin by the conversion of 7-dehydrocholesterol to cholecalciferol or vitamin $D_{3}$ under ultraviolet light exposure. Vitamin $D_{3}$ suffers sub-

\section{Corresponding author:}

Dr Vijay Kunadian MBBS, MD, FRCP, FACC, FESC, PGDip, Institute of Cellular Medicine, Faculty of Medical Sciences, Newcastle University, 3rd Floor William Leech Building, Newcastle upon Tyne, NE2 4HH, United Kingdom, phone: +44 (0) 1912085797 , fax: +44 (0) 191 242 4290, e-mail: vijay.kunadian@ncl.ac.uk

Received: 15.08.2015, accepted: 15.08.2015. 
sequent hydroxylation in the liver (25-hydroxyvitamin D) and finally in the kidneys, to produce its active form, 1,25-dihydroxyvitamin $\mathrm{D}\left[1,25(\mathrm{OH})_{2} \mathrm{D}\right]$. It exerts its function by binding to the vitamin $\mathrm{D}$ receptor (VDR), which is widely distributed in human tissues. Recently, Ni et al. [7] demonstrated that deletion of VDR, by using a specific endothelial VDR knockout mice model, resulted in decreased expression of endothelial nitric oxide (NO) synthase expression and impaired endothelial function. In addition, it has been shown that vitamin D promotes the proliferation and migration of human umbilical vein endothelial cells (HUVEC) in a 3D matrix in a NO-dependent fashion [8]. Nitric oxide and NO synthase are key mediators of vasculogenesis and angiogenesis, and crucial for arteriogenesis [9]. In addition to NO modulation, vitamin $D$ also seems to regulate other pathways involved in endothelial repair (Figure 1). Endothelial progenitor cells treated with $10 \mathrm{nM}$ vitamin $\mathrm{D}$ in vitro presented increased proliferation, length of tubule formation on Matrigel and VEGF mRNA levels, indicating that vitamin $D$ improves the angiogenic properties of such cells via regulation of the VEGF signalling pathway [10]. Also, vitamin $\mathrm{D}$ was shown to promote vascular regeneration. Vitamin D treatment (100 ng/kg per day) of mice subject- ed to electric injury of carotid arteries promoted re-endothelialization and augmented the number of angiogenic myeloid cells (AMC). In diabetic mice whose femoral artery was ligated, treatment with vitamin D also promoted re-endothelialization and restored angiogenesis. In 6 healthy volunteers ( 4 male; 2 female; mean age \pm SD: $32.5 \pm 4.9$ years) treated orally with 4000 IU of vitamin $D_{3}$ supplement per day for 6 weeks, the number of circulating myeloid cells increased in comparison to baseline levels. The pro-angiogenic effect of vitamin D was associated with its action on AMC, which increases the levels of stromal cell-derived factor 1 (SDF1) in the plasma and injured tissues [11]. Although there is evidence supporting a positive modulation of vitamin D over both arteriogenesis and angiogenesis, its association with coronary collateral circulation has only been investigated by a few clinical studies.

In this issue of Postępy w Kardiologii Interwencyjnej/ Advances in Interventional Cardiology, an association between circulating levels of 25 -hydroxyvitamin D and the degree of coronary collateral circulation development is reported. This study included 173 patients (mean age \pm SD: $65 \pm 11$ years, 120 men) with coronary total occlusion (СТO) in at least one artery without history of acute

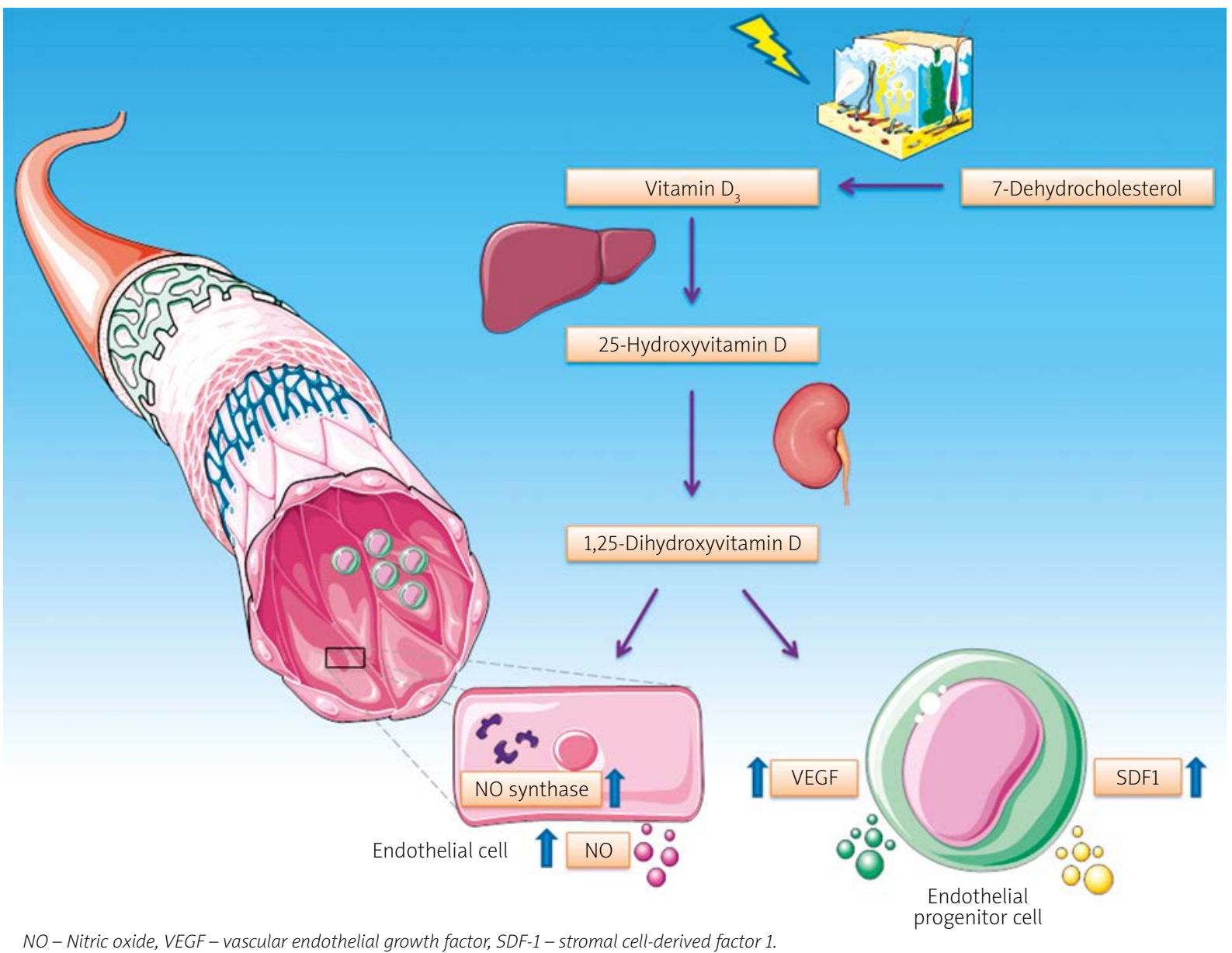

Figure 1. Pro-angiogenic effects of 1,25-dihydroxyvitamin D on endothelial cells and endothelial progenitor cells 
coronary syndromes in the past 3 months. The presence of collaterals was assessed by coronary angiography and the Rentrop classification was used to grade collateral circulation development. The authors observed that the group of patients with poorly developed collateral circulation (Rentrop 0 and 1 ) had significantly lower levels of vitamin D (mean \pm SD: $20 \pm 3 \mathrm{ng} / \mathrm{ml}$ ) in comparison with the group that had well-developed collaterals (Rentrop 2 and 3; mean \pm SD: $30 \pm 6 \mathrm{ng} / \mathrm{ml}$ ), $p<0.0001$. In addition, after multivariate regression analysis, vitamin $D$ levels were found to be an independent predictor of coronary collaterals development. This is one of the first studies to show such an association, especially amongst a cohort of patients presenting with CTO.

Previously, Sahin et al. [12], evaluating coronary collateral circulation in 214 patients with CAD by angiography, had reported lower levels of vitamin D in patients with Rentrop 0 and 1 in comparison with those classified as Rentrop 2 and 3 (mean \pm SD: $34 \pm 25$ pmol// vs. 49 $\pm 33 \mathrm{pmol} / \mathrm{l} ; p=0.01$ ). Vitamin $\mathrm{D}$ levels were also an independent predictor of collateral circulation development. Interestingly, Hossein-Nezhad et al. [13] found that a vitamin $D$ receptor gene polymorphism (Fokl) was associated with the degree of collateralization in 760 individuals undergoing coronary angiography due to suspected CAD. The majority (80.2\%) of those homozygous for the $\mathrm{F}$ allele (FF genotype) were in the well-developed collateral circulation group as compared to $51.72 \%$ of those homozygous for the $f$ allele (ff genotype), $p=0.01$. The $\mathrm{F}$ allele codes a VDR sequence which is 3 amino acids shorter in length than the fallele, which could imply different functional properties of the VDR receptor.

A significant limitation of such studies is the method used for assessment of coronary collateral vessels, i.e. coronary angiography. This technique is not able to detect collaterals $<200 \mu \mathrm{m}$ in diameter, which account for most of these vessels. It seems that invasive measurement of flow and pressure - such as the CFI - is the current gold standard for assessment of coronary collaterals' functional significance, as it provides more direct, quantitative results and is a good predictor of ischemia and future ischemic events [14]. Also, it is not known whether the association of lower vitamin $D$ levels and poor coronary collateral circulation is causal. Whilst data from several clinical studies associate vitamin D deficiency with increased CAD burden and worse cardiovascular outcomes, the findings from the few randomized clinical trials that evaluated vitamin D supplementation have failed to demonstrate a significant effect on cardiovascular events and surrogate markers of CAD. In addition, vitamin D deficiency has been correlated with frailty, and it is known that physical exercise promotes the development of coronary collaterals. Thus, this could be a confounding variable when assessing the relationship between vitamin D levels and development of collaterals [15].
In conclusion, vitamin D seems to play an important role in the regulation of key mechanisms involved in coronary collateral circulation development. There is already some clinical evidence of a correlation between circulating levels of vitamin D and collateral presence; however, studies assessing the coronary collateral circulation function using more reliable invasive techniques are necessary to corroborate this association.

\section{Conflict of interest}

The authors declare no conflict of interest.

\section{References}

1. Maxwell MP, Hearse DJ, Yellon DM. Species variation in the coronary collateral circulation during regional myocardial ischaemia: a critical determinant of the rate of evolution and extent of myocardial infarction. Cardiovasc Res 1987; 21: 737-46.

2. Carmeliet P. Mechanisms of angiogenesis and arteriogenesis. Nat Med 2000; 6: 389-95.

3. Seiler C. Collateral circulation of the heart. Springer, London 2009.

4. Werner GS, Ferrari M, Betge S, et al. Collateral function in chronic total coronary occlusions is related to regional myocardial function and duration of occlusion. Circulation 2001; 104: 2784-90.

5. Seiler C, Engler R, Berner L, et al. Prognostic relevance of coronary collateral function: confounded or causal relationship? Heart 2013; 99: 1408-14.

6. Meier P, Hemingway $\mathrm{H}$, Lansky AJ, et al. The impact of the coronary collateral circulation on mortality: a meta-analysis. Eur Heart J 2012; 33: 614-21.

7. Ni W, Watts SW, Ng M, et al. Elimination of vitamin D receptor in vascular endothelial cells alters vascular function. Hypertension 2014; 64: 1290-8

8. Pittarella P, Squarzanti DF, Molinari C, et al. NO-dependent proliferation and migration induced by vitamin D in HUVEC. J Steroid Biochem Mol Biol 2015; 149: 35-42.

9. Cai WJ, Kocsis E, Luo X, et al. Expression of endothelial nitric oxide synthase in the vascular wall during arteriogenesis. Mol Cell Biochem 2004; 264: 193-200.

10. Grundmann M, Haidar M, Placzko S, et al. Vitamin D improves the angiogenic properties of endothelial progenitor cells. Am J Physiol Cell Physiol 2012; 303: C954-62.

11. Wong MS, Leisegang MS, Kruse C, et al. Vitamin D promotes vascular regeneration. Circulation 2014; 130: 976-86.

12. Sahin I, Okuyan E, Gungor B, et al. Lower vitamin D level is associated with poor coronary collateral circulation. Scand Cardiovasc J 2014; 48: 278-83.

13. Hossein-Nezhad A, Eshaghi SM, Maghbooli Z, et al. The role of vitamin $D$ deficiency and vitamin $D$ receptor genotypes on the degree of collateralization in patients with suspected coronary artery disease. Biomed Res Int 2014; 2014: 304250.

14. Khand A, Fisher M, Jones J, et al. The collateral circulation of the heart in coronary total arterial occlusions in man: systematic review of assessment and pathophysiology. Am Heart J 2013; 166: 941-52.

15. Kunadian V, Ford GA, Bawamia B, et al. Vitamin D deficiency and coronary artery disease: a review of the evidence. Am Heart J 2014; 167: 283-91. 